\title{
Aspergillosis in Immunocompetent Women: Challenging Diagnosis in The Intensive Care Unit
}

\author{
Aragão J0, Pinheiro DOBP and Montenegro FS* \\ Internal Medicine Residency Program, Adventist Silvestre Hospital, Rio de Janeiro, Brasil
}

Received: 眥 January 08, 2019; Published: 眥January 17, 2019

*Corresponding author: Fernando Santiago Montenegro, Internal Medicine Residency Program, Adventist Silvestre Hospital, Rio de Janeiro, Brasil

\begin{abstract}
Aspergillosis is a systemic mycosis caused by Aspergillus spp whose invasive form is manifested particularly in immunocompromised patients. In the last decade, reports have documented the expansion of invasive aspergillosis beyond neutropenic patients including patients admitted to the intensive care unit. In this setting clinical diagnostic is challenging due to the low incidence, lack of suspicion and unvalidated diagnostic definitions. We present a case of an elderly woman with bronchiectasis and respiratory failure whose broad-spectrum antibiotic treatment was not effective and whose diagnostic tests suggested invasive aspergillosis. The purpose of this report is to draw attention to the need for high clinical suspicion of invasive fungal infection within intensive care patients, particularly those with risk factors or not responsive to the initial antimicrobial therapy.
\end{abstract}

Abbreviations: BAL: Bronchoalveolar Lavage; COPD: Chronic Pulmonary Disease; IA: Invasive Aspergillosis; ICU: Intensive Care Unity; PCR: Polymerase Chain Reaction

\section{Introduction}

Aspergillosis is a systemic mycosis caused by Aspergillus spp. They are ubiquitous filamentous fungi found especially in the soil, with decaying matter [1]. They were first described during prolonged neutropenia and most cases of invasive aspergillosis (IA) are seen in the immunocompromised hosts [2]. They can cause broadly spectrum of disease whose main manifestations are expressed in the respiratory tract [3]. A. fumigatus is the main agent of invasive aspergillosis, and chronic and allergic forms [4]. The fungus can colonize the respiratory mucosa even of immunocompetent people. However, the invasive form is manifested particularly in immunocompromised patients, most notable in chronic neutropenic patients [5]. Besides the immunocompromised setting, IA can be seen in the intensive care unit (ICU) in patients who do not meet the above criteria [6]. Incidence rates ranges from $0,3 \%$ to $5,8 \%$ with mortality rates over $80 \%$ [7]. Delsuc et al. in a cohort study in two university hospital ICUs found that IA was a strong death predictor in critically ill patients with chronic obstructive pulmonary disease (COPD). The use of corticosteroids and antibiotics before ICU admission were also a risk factor for IA [8]. The invasive form can be divided in angioinvasive, bronchopulmonary, or chronic necrotizing manifestations. Nonspecific clinical manifestations and radiological patterns, and the limited value of mycological criteria makes diagnostic of this disease challenging in the ICU setting confirming the high mortality rate of this disease.

\section{Case Report}

An 81 years-old woman was admitted in the emergency department with progressive dyspnea, cough, and fever started four days before. She had systemic hypertension, hypothyroidism, and a history of previous bronchiectasis and had treated a communityacquired pneumonia three months before with amoxicillinclavulanate. At admission, she had a severe bronchospasm and respiratory insufficiency, needing prompt orotracheal intubation. A thoracic computed tomography was performed showing centrilobular nodules configuring tree-in-bud aspect, and air-fluid level lesion at inferior left lobe (Figure 1). Antibiotic therapy was initiated with piperacillin-tazobactam and clarithromycin. Initial laboratory exams showed leukocytosis $\left(13,930 / \mathrm{mm}^{3}\right)$ and elevated C-reaction protein $(4,1 \mathrm{mg} / \mathrm{dL})$. She was admitted to the ICU under midazolam sedation and dependent of vasoactive drug therapy. Her APACHE II score was 25. At the fifth day of hospitalization, despite broad spectrum antibiotic the patient presented with worsening of leukocytosis and inflammatory markers, as well acute renal injury, being submitted to dialysis. Due to the lack of clinical improvement a bronchoscopy was performed showing friable respiratory mucosa. 


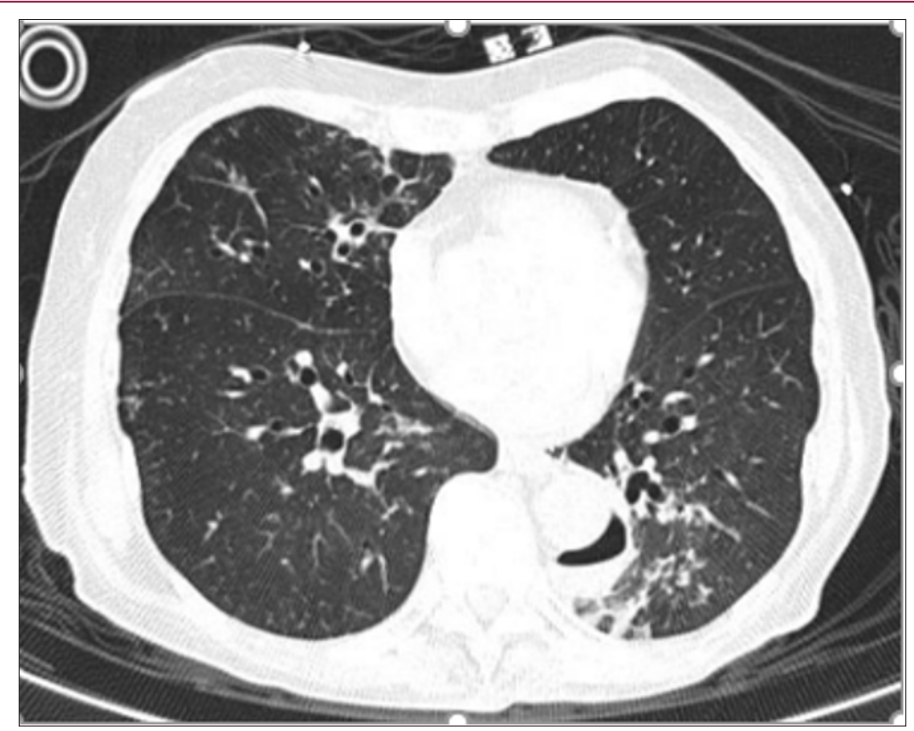

Figure 1: Patient's computed tomography showing a tree-in-bud aspect and a cavity.

Bronchoalveolar lavage (BAL) material was taken for analysis. The antimicrobial scheme was changed to meropenem and moxifloxacin. As the patient after 48 hours remained clinically unstable the antibiotic regime was reviewed and antifungal coverage with amphotericin B was associated. Despite treatment the patient died 10 days after admission. Postmortem, the BAL exams exhibited positive culture for Stenotrophomonas maltophilia and a positive search for galactomannan antigen. Serologic exams for blastomycosis, histoplasmosis, and cryptococcosis were all negative. Exams for tuberculosis were also negative (including polymerase chain reaction [PCR] test). Specific culture for mycobacteria and fungi were also negative.

\section{Discussion}

This case describes an immunocompetent woman with pulmonary structural alterations, with a worsening pulmonary infection evolution, although broad spectrum antibiotic treatment. The antifungal treatment was added late in the course of the disease which prevented her from having the expected response. The lack of clinical manifestations and the difficult diagnosis led to a delay initiation of antifungal treatment. In the work of Desluc et al. the median delay of antifungal treatment initiation was 8 days after ICU admission and 4 days after Aspergillus spp.- positive lower respiratory tract specimen [8] which was the same as in our case. He identified three variables that were independent predictors of invasive bronchial-pulmonary aspergillosis before ICU admission: more than three kinds of antibiotics used, accumulated doses of corticosteroids ( $>350 \mathrm{mg}$ ) and APACHE II scores $>18$ [9]. Our patient had an APACHE score of 25, placing her at higher risk for IA. Laboratorial findings concluded for a positive quantitative galactomannan antigen. However, piperacillin-tazobactam could cause false-positive result for galactomannan antigen test. Nevertheless, currently guidelines do not support this relation [10-11]. Galactomannan antigen from BAL is considered to have sensibility of $87 \%$ e specificity of $89 \%$ [12].
A systematic review showed a sensitivity and specificity of 77\% and 94\%, respectively, considering PCR of BAL [13]. Radiological findings can be non-specific especially in nonneutropenic patients. Computed tomography images like centrilobular nodule, tree-inbud aspect, ground-glass opacities, cavitating lesions, and others do not give a $100 \%$ sensitivity or specificity. In retrospective analyses, Dai et al. observed that the most frequently CT abnormality was infiltrates and only $8.3 \%$ of the patients showed the air crescent sign, and none showed the halo sign [14]. Treatment with voriconazole is the first line antifungal agent for aspergillosis, but other agents can be used, and amphotericin B in critically ill patients is recommended [15]. In summary, critically ill patients are at risk of invasive fungal infections, especially those with pulmonary structural alterations and lack of response to broadly antibacterial regime. High level clinical suspicion is important when considering extending the antimicrobial treatment for covering fungus. A validated diagnostic algorithm may be helpful in the ICU department to identify patients who are at high risk of IA and in whom antifungal treatment should be started earlier.

\section{References}

1. Kousha M, Tadi R, Soubani AO (2011) Pulmonary aspergillosis: a clinical review. Eur Respir Rev 20(121): 156-174.

2. Patterson TF, Kirkpatrick WR, White M, Hiemenz JW, Wingard JR, et al. (2000) Invasive aspergillosis: disease spectrum, treatment practices, and outcomes. Medicine 79(4): 250-260.

3. Kosmidis C, Denning DW (2015) The clinical spectrum of pulmonary aspergillosis. Thorax 70(3): 270-277.

4. Chabi ML, Goracci A, Roche N, Paugam A, Lupo A, et al. (2015) Pulmonary aspergillosis. Diagn Interv Imaging 96: 435-442.

5. Auberger J, Lass Flörl C, Ulmer H, Nogler Semenitz E, Clausen J, et al. (2008) Significant alterations in the epidemiology and treatment outcome of invasive fungal infections in patients with hematological malignancies. Int J Hematol 88(5): 508-515.

6. Meersseman W, Vandecasteele SJ, Wilmer A, Verbeken E, Peetermans WE, et al. (2004) Invasive aspergillosis in critically ill patients without malignancy. Am J Respir Crit Care Med 170(6): 621-625. 
7. Taccone FS, Van den Abeele AM, Bulpa P, Misset B, Meersseman W, et al. (2015) Epidemiology of invasive aspergillosis in critically ill patients: clinical presentation, underlying conditions, and outcomes. Crit Care 19: 7.

8. Delsuc C, Cottereau A, Frealle E, Bienvenu AL, Dessein R, et al. (2015) Putative invasive pulmonary aspergillosis in critically ill patients with chronic obstructive pulmonary disease: a matched cohort study. Crit Care 19: 421 .

9. He H, Ding L, Li F, Zhan Q (2011) Clinical features of invasive bronchialpulmonary aspergillosis in critically ill patients with chronic obstructive respiratory diseases: a prospective study. Crit Care 15(1): 5.

10. Ullmann AJ, Aguado JM, Arikan Akdagli S, Denning DW, Groll AH, et al. (2018) Diagnosis and management of Aspergillus diseases: executive summary of the 2017 ESCMIDECMM-ERS guideline. Clin Microbiol Infect 24(1): 1-38.

11. Patterson TF, Thompson GR, Denning DW, Fishman JA, Hadley S, et al. (2016) Practice Guidelines for the Diagnosis and Management

ISSN: 2574-1241

DOI: 10.26717/BJSTR.2019.13.002369

Montenegro FS. Biomed J Sci \& Tech Res

(c) (i) This work is licensed under Creative

Submission Link: https://biomedres.us/submit-manuscript.php of Aspergillosis: 2016 Update by the Infectious Diseases Society of America. Clin Infect Dis 63(4): 1-60.

12. Zou M, Tang L, Zhao S, Zhao Z, Chen L, et al. (2012) Systematic review and meta-analysis of detecting galactomannan in bronchoalveolar lavage fluid for diagnosing invasive aspergilosis. PLoS ONE 7(8): 43347.

13. Avni T, Levy I, Sprecher H, Yahav D, Leibovici L, et al. (2012) Diagnostic accuracy of PCR alone compared to galactomannan in bronchoalveolar lavage fluid for diagnosis of invasive pulmonary aspergillosis: a systematic review. J Clin Microbiol 50(11): 3652-3658.

14. Dai Z, Zhao H, Cai S, LV Y, Tong W, et al. (2013) Invasive pulmonary aspergillosis in nonneutropenic patients with and without underlying disease: a single-centre retrospective analysis of 52 subjects. Respirology 18(2): 323-331.

15. Herbrecht R, Denning DW, Patterson TF, Bennet JE, Greene RE, et al. (2002) Voriconazole versus amphotericin B for primary therapy of invasive aspergillosis. N Eng J Med 347(6): 408-415.



